\title{
Semi-Automated Registration of 3D Torso Images from Breast Reconstruction Surgery
}

\author{
Lijuan $\mathrm{ZHAO}^{1}$, Shishir K. SHAH ${ }^{1}$, Gregory P. REECE ${ }^{2}$, Melissa A. CROSBY ${ }^{2}$, Elisabeth K. BEAHM ${ }^{2}$, \\ Michelle C. FINGERET ${ }^{3}$, Mia K. MARKEY ${ }^{4,5}$, Fatima A. MERCHANT ${ }^{* 1,6}$ \\ ${ }^{1}$ Department of Computer Science, University of Houston, Houston (TX), USA; \\ ${ }^{2}$ Dept. of Plastic Surgery, The University of Texas MD Anderson Cancer Center, Houston (TX), USA; \\ ${ }^{3}$ Dept. of Behavioral Science, The University of Texas MD Anderson Cancer Center, Houston (TX), USA; \\ ${ }^{4}$ Department of Biomedical Engineering, The University of Texas at Austin, Austin (TX), USA; \\ ${ }^{5}$ Dept. of Imaging Physics, The University of Texas MD Anderson Cancer Center, Houston (TX), USA; \\ ${ }^{6}$ Department of Engineering Technology, University of Houston, Houston (TX), USA
}

\begin{abstract}
The applications for three-dimensional (3D) scanners in breast reconstruction surgery, which is a multi-step process lasting at times 2-3 years are increasing. In order to quantify changes occurring in the reconstructed breasts over time, it is necessary to register images acquired from patients during multiple clinical visits. In this study we present a semi-automated approach that allows registration of $3 \mathrm{D}$ images such that the differences in image-coordinate systems are removed, while optimizing correspondence of the breasts. Achieving this initial correspondence is essential to quantify differences between the two surfaces and to record the local morphological changes in the breast.
\end{abstract}

Keywords: 3D image, torso matching, 3D rigid registration, breast reconstruction, and breast morphology

\section{Introduction}

Breast reconstruction is an important surgical component for many women undergoing breast cancer treatment [1]. The purpose of breast reconstruction is to recreate a breast form that is satisfying to the patient, facilitating her psychosocial adjustment to living as a breast cancer survivor [2]. The term "breast reconstructive surgery" actually encompasses a range of surgeries performed immediately after mastectomy, or as separate procedures at a later date. The reconstructed breast can be formed using a breast tissue expander/implant, autologous tissue (i.e., living tissue from another part of the patient), or a combination of the two. In addition, surgical modifications are typically made to both breasts, even if only one breast had cancer, in order to maintain a symmetrical appearance. Thus, breast reconstruction is a complex process usually not achieved in a single procedure, and can take up to a year or longer, depending on the type of reconstructive procedures selected and other factors affecting surgical outcome, including postoperative complications and adjuvant cancer treatment. Recently, 3D imaging is increasingly utilized as a tool for quantitative breast morphometry [3-7]. In order to quantitate changes in breast morphology occurring during the time course of several reconstructive procedures, one needs to analyze 3D torso images acquired at successive time points from patients undergoing the multiple surgeries of their breast reconstructive course. In addition to breast surgery related anatomical changes (see Fig. 1 and Fig. 2), the multi-visit images for a patient may also change as a result of variations in the (1) object coordinate systems due to differences in patient positioning and posture; and (2) patient's BMI due to physiological weight changes. As a first step, registration of the multi-visit images is thus required in order to monitor and quantify the morphological changes occurring in the breasts.

Image registration is a process for determining the correspondence of features between images collected at different times or using different imaging modalities [8]. Our goal is to use the correspondences achieved between the multi-visit images to view and analyze images in the same coordinate system, and to facilitate quantification of morphological changes in breast appearance over time in the individual. Image registration methods are broadly classified as "rigid" (where images are assumed to be of objects that simply need to be rotated and translated with respect to one another to achieve correspondence) or "non-rigid" (where either through biological differences or image acquisition or both, correspondence between structures in two images cannot be achieved without some localized stretching of the images). The mapping of one 3D surface has been extensively studied 
in computer graphics and depending on the driving application, many different automated, semi-automated, and interactive approaches exist for registering two surfaces with each other [8,9]. The Iterative Closest Point (ICP) algorithm is most commonly used for registration [10-12], and for the integration of multiple range images for generating a surface model $[13,14]$. ICP methods determine a set of rigid motion parameters that register a range image to a given mesh-based geometric model. These algorithms typically do not require extracted features, curves or surface derivatives, except for the removal of statistical outliers like noise and occlusion. Although ICP methods are efficient and robust for 3D model reconstruction from multiple object views, in the case of mapping multi-visit images of breast reconstruction they may not achieve acceptable surface matching as the areas of arms and regions of breasts may have large deformities due to pose and surgical procedures, respectively. Most methods for non-rigid medical image registration rely on features that are extracted from images. For instance, Sun et. al, generate a 2D point fingerprint by projecting geodesic circles on the tangent plane [15]. Corresponding points on surfaces from different images are then found by comparing their fingerprints. Zou et. al, developed a salient point-based shape description wherein key surface points are extracted from the images at the geodesic scale and used to achieve correspondence [16]. These saliency-driven key points were computed as local extrema of the difference of Gaussian function defined over a curved surface in geodesic scale space to achieve 3D correspondence. Similarly, Wen suggested a medical image registration method using points, contours and curves [17]. The features were extracted from the images using a semi-automatic algorithm. Zeng et. al, proposed a high-order graph matching formulation to address surface matching, wherein singleton terms were used to capture the geometry, curvature and texture information while high-order terms were used to model the intrinsic embedding energy [18]. All these methods extract features from image areas where salient changes are observed. In this study we focus on multi-visit 3D image registration, wherein our goal is to match the $3 \mathrm{D}$ torsos along the chest wall which is anatomically relatively flat and does not exhibit salient features that can be extracted. Although anatomically the breast mounds exhibit characteristic morphology, such as nipples that can be used as features for surface mapping, in this study the multi-visit images differ in the region of the breast mounds due to surgical deformations thereby eliminating the possibility of using algorithms that rely on features extracted from images. We thus approach the problem using a rigid registration algorithm as described below.

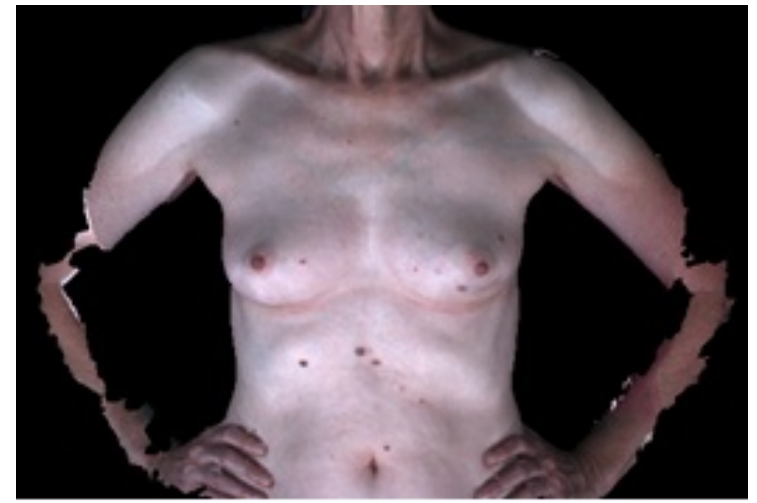

Fig. 1. Surface scan from previous visit (pre-operative).

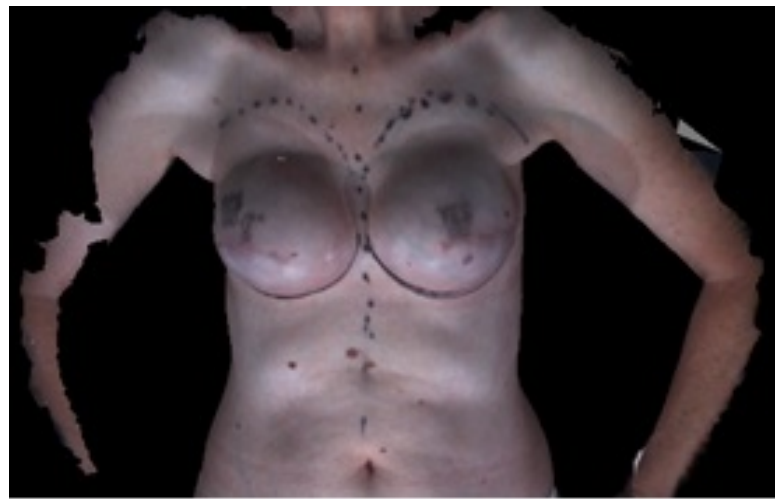

Fig. 2. Surface scan from latter visit (post-operative).

\section{Method}

The objective of this study was to develop an algorithm for the semi-automated registration of 3D torso images from multiple visits for the same patient, in order to quantify the morphological changes in 3D torso images of the same patient over time, due to differences in object coordinate system, patient weight and position, and surgical intervention. Our approach relies on the assumption that while the soft tissues of the patient's body may change over time, the skeleton is relatively stable. Thus the skeletal frame can be treated as being rigid, i.e. involving only translational and rotational transformations. Selecting points with reference to the skeletal frame and maximizing the correspondence between these points can then achieve 3D image registration. 


\subsection{Fiducial and Reference Points Selection}

First, we manually identify two fiducial points: sternal notch (SN) and umbilicus (UM). Next, thirteen reference points are automatically selected in the $y$ direction along a vertical (midline) axis and in the $x$ direction along horizontal axes perpendicular to the midline, at predefined distances from the two fiducial points giving a total of 15 points (see Fig. 3). To improve precision in the automatic selection of the reference points, we initially adjust the surface image to be symmetric about the $Y Z$ plane and use interpolation to compute the coordinates of the reference points as described in the following two sections.

\subsubsection{Surface alignment for selection of reference points}

The 3D images acquired from participants typically vary from the forward facing upright position (i.e. alignment along the $\mathrm{X}$ and $\mathrm{Y}$-axis) ranging anywhere from $0-90^{\circ}$ due to variations in patient positioning across the multiple clinical visits. These variations tend to introduce large discrepancies in the automatically selected reference points. To mitigate this effect, we initially align the medial axis of the torso along the line joining SN-UM to be coincident with $\mathrm{Y}$-axis and the 3D torso to be symmetric about YZ plane as follows.

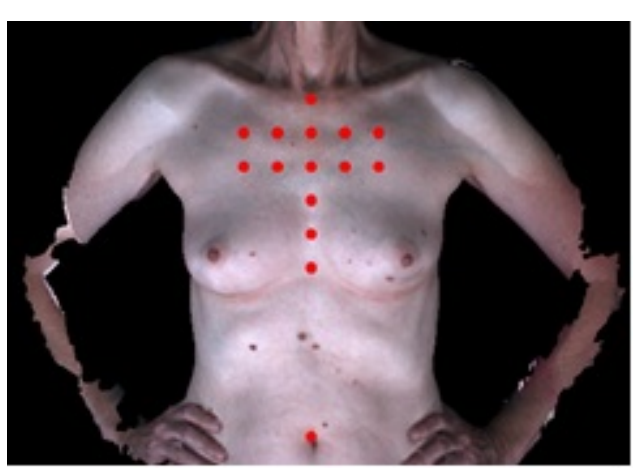

Fig. 3. Reference points selected on the torso for $3 D$ correspondence.

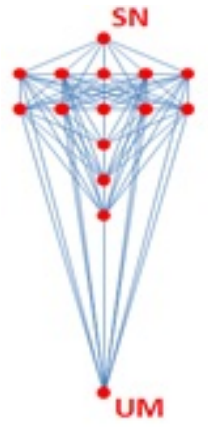

Fig. 4. Distance graph illustrating the selected reference points and the distances computed between the points and the two fiducial landmarks (SN and UM).

First, we transform the image such that $\mathrm{SN}$ and UM have the same $x$ coordinate, i.e., the image is translated such that the $\mathrm{SN}$ is at the origin and then rotated about the Z-axis. The angle of rotation about the Z-axis and the transformation matrix are given as:

$$
\gamma=\sin ^{-1}\left(\frac{-x_{2}}{\sqrt{x_{2}^{2}+y_{2}^{2}}}\right) \text { and }\left[\begin{array}{cccc}
1 & 0 & 0 & 0 \\
0 & 1 & 0 & 0 \\
0 & 0 & 1 & 0 \\
-x_{1} & -y_{1} & -z_{1} & 1
\end{array}\right]\left[\begin{array}{cccc}
\cos \gamma & \sin \gamma & 0 & 0 \\
-\sin \gamma & \cos \gamma & 0 & 0 \\
0 & 0 & 1 & 0 \\
0 & 0 & 0 & 1
\end{array}\right]
$$

where $x_{1}, y_{1}, z_{1}$ represent the $x, y$, and $z$ coordinates of $\mathrm{SN}$ (before translation); $x_{2}$ and $y_{2}$ represent the $x$ and $y$ coordinates of the UM (after translation).

Next, we rotate image about the $\mathrm{X}$-axis such that medial axis along SN-UM coincides with the $\mathrm{Y}$-axis. The angle of rotation about the $\mathrm{X}$-axis and the transformation matrix are:

$$
\alpha=\sin ^{-1}\left(\frac{z_{2}^{\prime}}{\sqrt{{y_{2}^{\prime}}^{2}+z_{2}^{\prime 2}}}\right) \text { and }\left[\begin{array}{cccc}
1 & 0 & 0 & 0 \\
0 & \cos \alpha & \sin \alpha & 0 \\
0 & -\sin \alpha & \cos \alpha & 0 \\
0 & 0 & 0 & 1
\end{array}\right]
$$

where $y_{2}^{\prime}$ and $z_{2}^{\prime}$ represent the $y$ and $z$ coordinates of UM (following rotation about the Z-axis in the previous step).

Finally, we rotate the image about the $\mathrm{Y}$-axis to achieve surface symmetry about the $\mathrm{YZ}$ plane. Two symmetric points $S_{r}\left(-x_{m}, y_{m}, z_{r}\right)$ and $S_{l}\left(x_{m}, y_{m}, z_{l}\right)$ are determined on the surface based on the predefined $x_{m}$ and $y_{m}$ values. $z_{r}$ and $z_{l}$ are calculated using the interpolation method described below in section 2.1.2. Let two vectors $\vec{a}$ and $\vec{b}$ be the projections for vectors $\overrightarrow{O S_{r}}$ and $\overrightarrow{O S_{l}}$ on the $\mathrm{XZ}$ plane, then $\vec{a}=\left(-x_{m}, 0, z_{r}\right)$ and $\vec{b}=\left(x_{m}, 0, z_{l}\right)$. The internal angle bisector between $\vec{a}$ and $\vec{b}$ is 
defined as $\vec{c}=\frac{\vec{a}}{|\vec{a}|}+\frac{\vec{a}}{|\vec{a}|}$. The angle of rotation about the Y-axis and the transformation matrix are determined as:

$$
\beta=\tan ^{-1}\left(-\frac{x_{c}}{z_{c}}\right) \text { and }\left[\begin{array}{cccc}
\cos \beta & 0 & -\sin \beta & 0 \\
0 & 1 & 0 & 0 \\
\sin \beta & 0 & \cos \beta & 0 \\
0 & 0 & 0 & 1
\end{array}\right]
$$

where $x_{c}$ and $z_{c}$ represent the $x$ and $z$ components of $\vec{c}$.

\subsubsection{Coordinate Interpolation}

Since the initial manual selection of the fiducial points (SN and UM) may be subject to operator bias, we automatically select the additional thirteen reference points at predefined $x$ and $y$ coordinates as follows.

Let $d$ represent the straight line distance between the two fiducial points $\mathrm{SN}$ and $\mathrm{UM}$, the coordinates of the $i$ th reference point, $\left(x_{i}, y_{i}\right)$ are determined as:

$$
\begin{gathered}
\left(0,-\frac{d}{10}\right),\left(0,-\frac{2 d}{10}\right),\left(0,-\frac{3 d}{10}\right),\left(0,-\frac{4 d}{10}\right),\left(0,-\frac{5 d}{10}\right),\left(\frac{d}{10},-\frac{d}{10}\right),\left(-\frac{d}{10},-\frac{d}{10}\right), \\
\left(\frac{2 d}{10},-\frac{d}{10}\right),\left(-\frac{2 d}{10},-\frac{d}{10}\right),\left(\frac{d}{10},-\frac{2 d}{10}\right),\left(-\frac{d}{10},-\frac{2 d}{10}\right),\left(\frac{2 d}{10},-\frac{2 d}{10}\right), \text { and }\left(-\frac{2 d}{10},-\frac{2 d}{10}\right) .
\end{gathered}
$$

Interpolation is used to determine the $z_{i}$ coordinate for each of the thirteen- $i$ th reference point as follows. We first project the surface triangles of the 3D mesh (Fig. 5) into the XY plane, i.e., only $x$ and $y$ coordinates for the vertices of the triangles are considered. For all projected triangle points, we next determine the triangle that encloses the point $\left(x_{i}, y_{i}\right)$ as follows. In a $2 \mathrm{D}$ plane, if a point $\mathrm{D}$ is in a triangle $\triangle A B C$ (see Fig. 6), then the area of $\triangle A B C$ equals the sum of areas of $\triangle A B D, \triangle B C D$, and $\triangle A D C$. Otherwise, point $D$ is not in $\triangle A B C$. For a point $D$ that is enclosed in $\triangle A B C$, we determine $z_{i}$ using linear interpolation based on $3 \mathrm{D}$ coordinates of 3 vertices of the triangle and $x_{i}$ and $y_{i}$ values for point $D$.

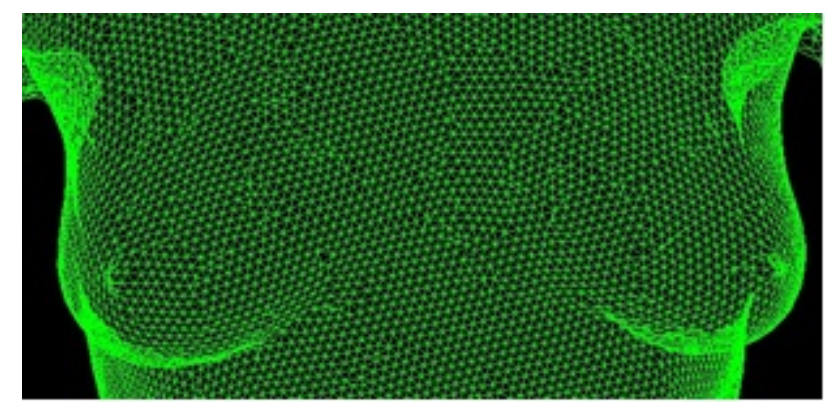

Fig. 5. Triangular surface mesh for 3D image.

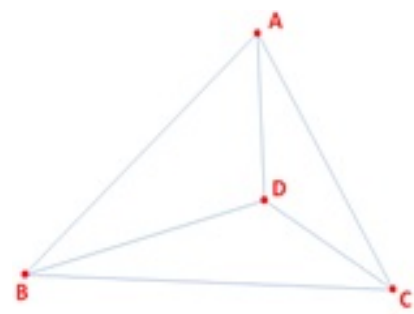

Fig. 6. A point enclosed within a triangle in a $2 D$ plane.

\subsection{Rigid Registration}

Images from two visits are registered by determining the parameters for rigid transformation as follows. 3D correspondence between fifteen points from two images, including the two fiducial landmarks (SN and $\mathrm{UM})$ and the thirteen reference points is achieved by optimizing six parameters $\left(\theta_{x}, \theta_{y}, \theta_{z}, t_{x}, t_{y}, t_{z}\right)$, where $\theta_{x}, \theta_{y}$, and $\theta_{z}$ are rotation angles about X-, Y-, and Z-axes, respectively; and $t_{x}, t_{y}, t_{z}$ are the displacements along $\mathrm{X}-, \mathrm{Y}-, \mathrm{Z}$-axes, respectively. Let $\left(x_{i}, y_{i}, z_{i}\right)$ and $\left(x_{i}^{\prime}, y_{i}^{\prime}, z_{i}^{\prime}\right)$ be the coordinates for the $i$ th point in the 3D images before and after transformation, then the transformed coordinates are determined as:

$$
\begin{aligned}
& {\left[\begin{array}{lll}
x_{i}^{\prime} & y_{i}^{\prime} & z_{i}^{\prime} \\
1
\end{array}\right]=}
\end{aligned}
$$

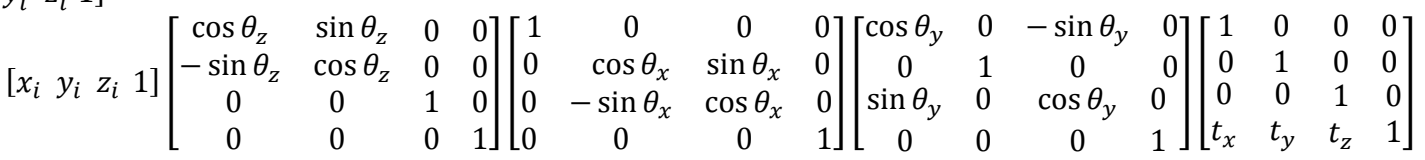


Fig. 4 illustrates the distance graph that is generated for each 3D image, by computing the pairwise distances between the thirteen reference points and the two fiducial landmarks. Each pair of points forms one edge. The total number of edges is $m=C_{n}^{2}$, where $n=15$. Images from two different visits are then registered by determining the parameters for rigid transformation using the following cost function:

$$
f=\sum_{i=1}^{n}\left[\left(x_{i 1}-x_{i 2}^{\prime}\right)^{2}+\left(y_{i 1}-y_{i 2}^{\prime}\right)^{2}+\left(z_{i 1}-z_{i 2}^{\prime}\right)^{2}\right]+\frac{2}{(n-1)} \sum_{j=1}^{m}\left(\theta_{j} * l\right)^{2}
$$

where $\left(x_{i 1}, y_{i 1}, z_{i 1}\right)$ and $\left(x_{i 2}^{\prime}, y_{i 2}^{\prime}, z_{i 2}^{\prime}\right)$ are the coordinates for the $i$ th point in the $3 \mathrm{D}$ image from the previous visit and latter visit (transformed) respectively, $\theta_{j}$ is the angle between $j$ th edges in previous visit and latter visit images, and $l$ is the average edge length for all edges in the two images. In the cost function, $\theta_{j} * l$ is the arc length. There are $n-1$ edges in the complete graph for each point and two points share one edge, so we normalize the second term by a coefficient $\frac{2}{(n-1)}$. The second term represents the arc length of the angle between the corresponding edges in two images. The transformation parameters are optimized when the cost function is minimized. We implemented the optimization of the cost function using the built-in optimization functions in Matlab (Mathworks, Natick, MA). Since the Matlab functions may return a local optimum value, we used two methods to find the global optima: (1) Implementation of an iterative optimization process, by using the result of the previous iteration as the initial value for the next iteration, and (2) Implementation of the large scale algorithm in Matlab for determining the next value during optimization, by computing the gradient, i.e., the partial differential for the 6 transformation parameters.

\subsection{Point Fingerprints}

We use a 3D surface representation scheme called point fingerprints to evaluate the results of our algorithm for achieving $3 \mathrm{D}$ registration. A point fingerprint is a set of $2 \mathrm{D}$ contours that are projections of geodesic circles onto a given tangent plane in local/global coordinate system [15]. In this study we use planes along Z-axis, which are parallel to $X Y$ plane to cut the surface to get a series of parallel circles. The point fingerprint contours are obtained by projecting the points of circles onto the XY plane. To compare the torso matching result, we put the point fingerprints of two related images into one 2D graph. We use the z-buffer algorithm to display overlapping points from the two point fingerprints, whereby if two points in different images project onto the same pixel, we plot the one with larger z-coordinate [19].

\section{Data Acquisition}

Female patients undergoing breast reconstruction surgery at the University of Texas MD Anderson Cancer Center were recruited under an Institutional Review Board (IRB) approved protocol. The 3D surface images were obtained using the 3DMDTorso system (3Q Technologies, Atlanta, GA). The reconstructed surface image consists of 3D positions $(x, y, z)$ and their corresponding color and texture information. Surfaces images from three patients with aged 54, 54 and 58, and BMI 25.9, 22.3 and 30.9 , respectively were used in this study. All three patients were white and not Hispanic/Latino.

\section{Results}

To demonstrate the accuracy of our algorithm, we present experimental results on the application of our algorithm to two cases: (1) Simulated images, wherein a 3D torso image is generated from an existing 3D image of a participant by computational rotation and translation in a predefined manner. These two images thus form a pair, wherein the surface mesh is topologically similar with the exception of the two images being in different coordinate systems (the corresponding points of the two images have different coordinates) due to the applied rotation and translation transformation applied to one. This pair of images is used to simulate images from two different visits wherein the co-ordinate geometry would differ, and (2) Multiple visit images from the same patient acquired at two different clinical visits. 


\subsection{Algorithm validation using simulated images}

We validated the proposed images using simulated images. A pair of images was used to simulate the two-visit scenario, wherein the second image was generated by transforming the first image (i.e. a sample patient image) using Eq. 4 above with the following parameters:

$$
\left(\theta_{x}, \theta_{y}, \theta_{z}, t_{x}, t_{y}, t_{z}\right)=(0.2,0.3,0.4,20,40,50)
$$

Fig. 7 shows the registration results for these two images. To facilitate viewing, the 3D torso images are cropped to display only the area of the upper torso encompassing the breast mounds. For the simulated image pair in Figure 7, the untransformed image (simulating visit 1 ) is shown in red, and the transformed image (simulating visit 2) is shown in blue. Figures 7A, 7C and 7E represent original data prior to 3D registration, and Figures 7B, 7D and 7F presents the results following implementation of the proposed algorithm. Figure $7 \mathrm{~A}$ and $7 \mathrm{~B}$ show the point clouds for the surface before and after registration, respectively. Figure $7 C$ and $7 D$ show the two fiducial points and 13 reference points for each of the two images in the pair (red represent visit 1 and blue represents visit 2) before and after registration, respectively. Finally, Figure $7 \mathrm{E}$ and $7 \mathrm{~F}$ present the $2 \mathrm{D}$ point fingerprints before and after $3 \mathrm{D}$ registration. As seen in Figure 7 (images in the left column), the original surfaces from visit1 (untransformed red) and visit2 (transformed; blue) are not matched. Following the implementation of the proposed registration algorithm, correspondence is achieved between the two surfaces (images in the right column). The residual of the cost function in the last step during optimization was found to be $2.53 \times 10^{-9} \mathrm{~mm}^{2}$.

\subsection{Algorithm evaluation using multiple-visit images from subjects}

Following algorithm validation using simulated images we tested the algorithm using two-visit images from subjects. Data from two patients are shown in Fig. 8 and Fig.9. Red is used to represents data from a previous visit (visit1) image and blue represents data from the latter visit (visit2) image. As seen in the figures, 3D correspondence was achieved as evident by the overlap of the chest walls. Note that the mismatches in the poses of the subjects can be visualized in the areas of the arms. Further the mismatches are noted in the region of the left breast in Fig. 9 due to the surgical deformations present. Following registration of the two-visit images shown in Fig. 8, the minimum value of the first term of the cost function in the last step during optimization was found to be $67.6 \mathrm{~mm}^{2}$, and that for the images in Fig. 9 was $50.7 \mathrm{~mm}^{2}$. We can roughly estimate the tolerance using these values as follows. The average for these two residuals is $59.15 \mathrm{~mm}^{2}$. It is the sum for the square distances for 15 corresponding points. Thus, the average tolerance for each pair of corresponding points is $1.99 \mathrm{~mm}$ totally and $1.15 \mathrm{~mm}$ in each direction ( $\mathrm{x}, \mathrm{y}$, and $\mathrm{z}$ ). This indicates that the surface images can be registered with a precision $<2 \mathrm{~mm}$ using the proposed algorithm.

\section{Conclusions}

We present an algorithm for the semi-automated rigid registration of $3 \mathrm{D}$ torso images from multiple-visits for the same patient. Selecting points with reference to the skeletal framework and maximizing the correspondence between these points using a rigid registration method achieves surface matching with a precision $<2 \mathrm{~mm}$. As expected, few mismatches between the registered images continue to persist due to the changes between the 3D surface images and due to variations in weight, surgical deformations, and pose changes. Moreover, manual identification of the two fiducial points $\mathrm{SN}$ and UM is subject to operator bias and may introduce artifacts in the registration due to discrepancies in the identification of these points in the 3D torso images from multiple visits.

\section{Acknowledgements}

This work was supported by a NIH grant 1R01CA143190-01A1. The authors recognize the support and contributions of patient data that were generously provided by David Chang, M.D., Mark Villa, M.D., and Roman Skoracki, M.D., of the Department of Plastic Surgery at The University of Texas MD Anderson Cancer Center for use in this study. 

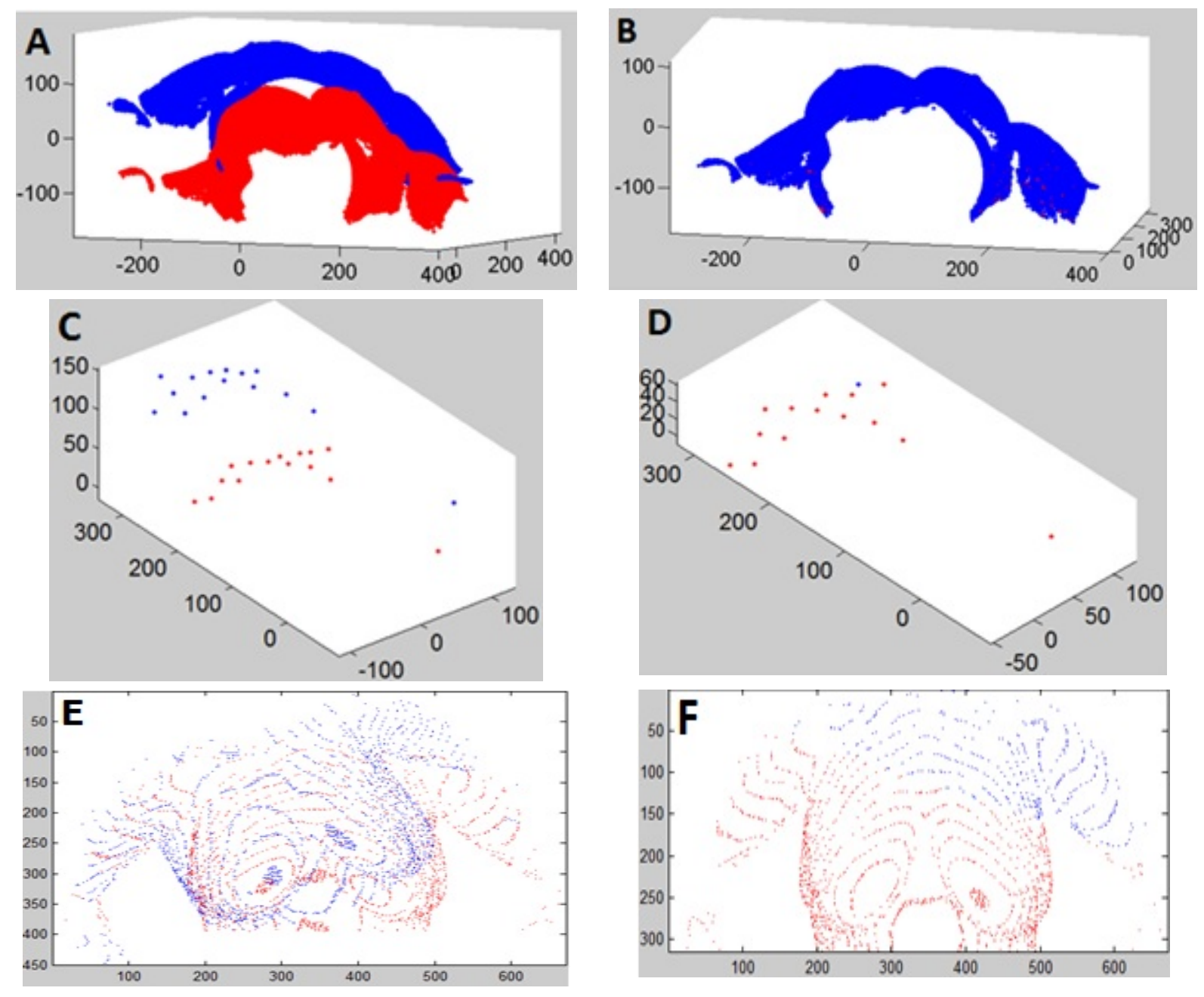

Fig. 7. Algorithm validation using simulated images (visit1 in red, visit2 in blue): (A) Original point clouds (bottom-up-view of breasts) showing unmatched surfaces, (B) 3D correspondence achieved following rigid transformation using the proposed algorithm, (C) Original fiducial points (SN and UM) and the 13 reference points, (D) $3 D$ correspondence achieved for fiducial points and selected points after registration, (E) $2 D$ point fingerprints for original surfaces, $(F) 2 D$ point fingerprint for surfaces after registration. 

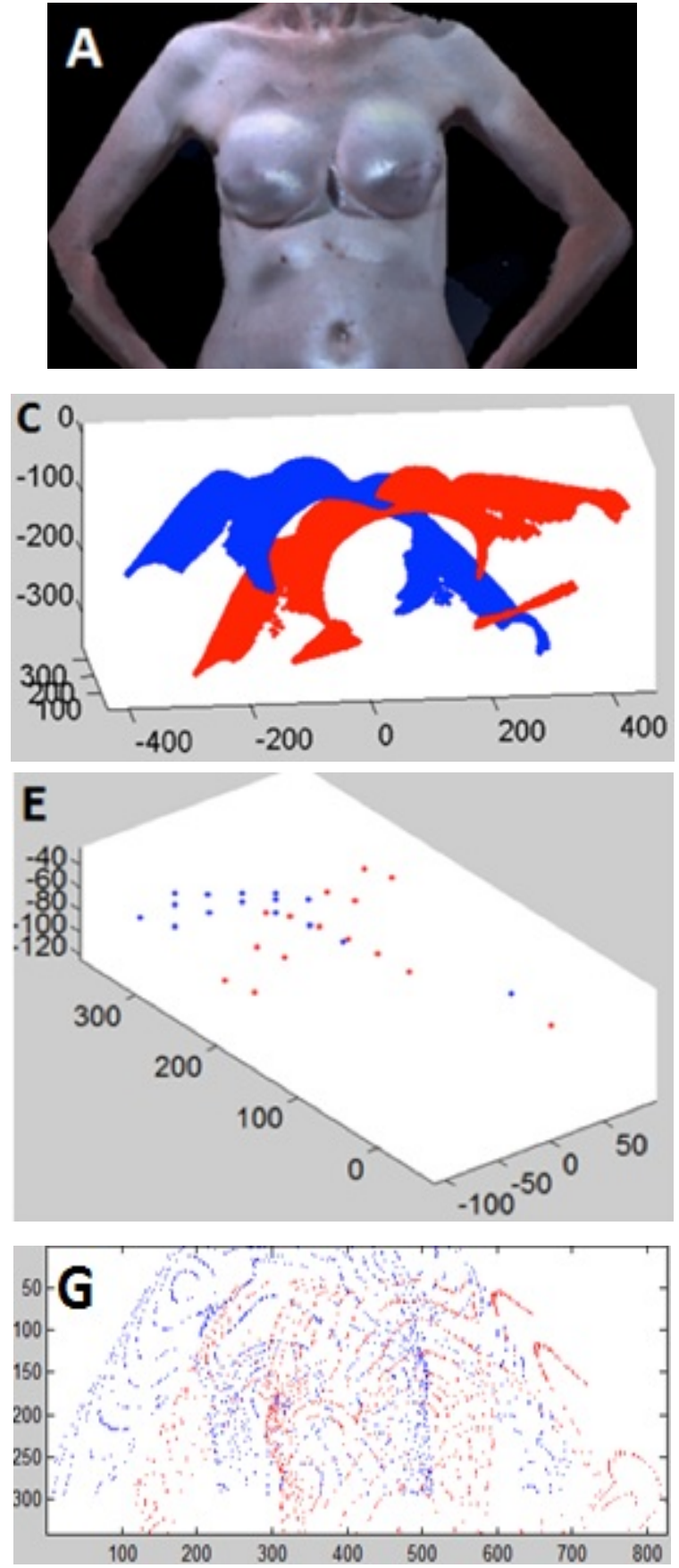
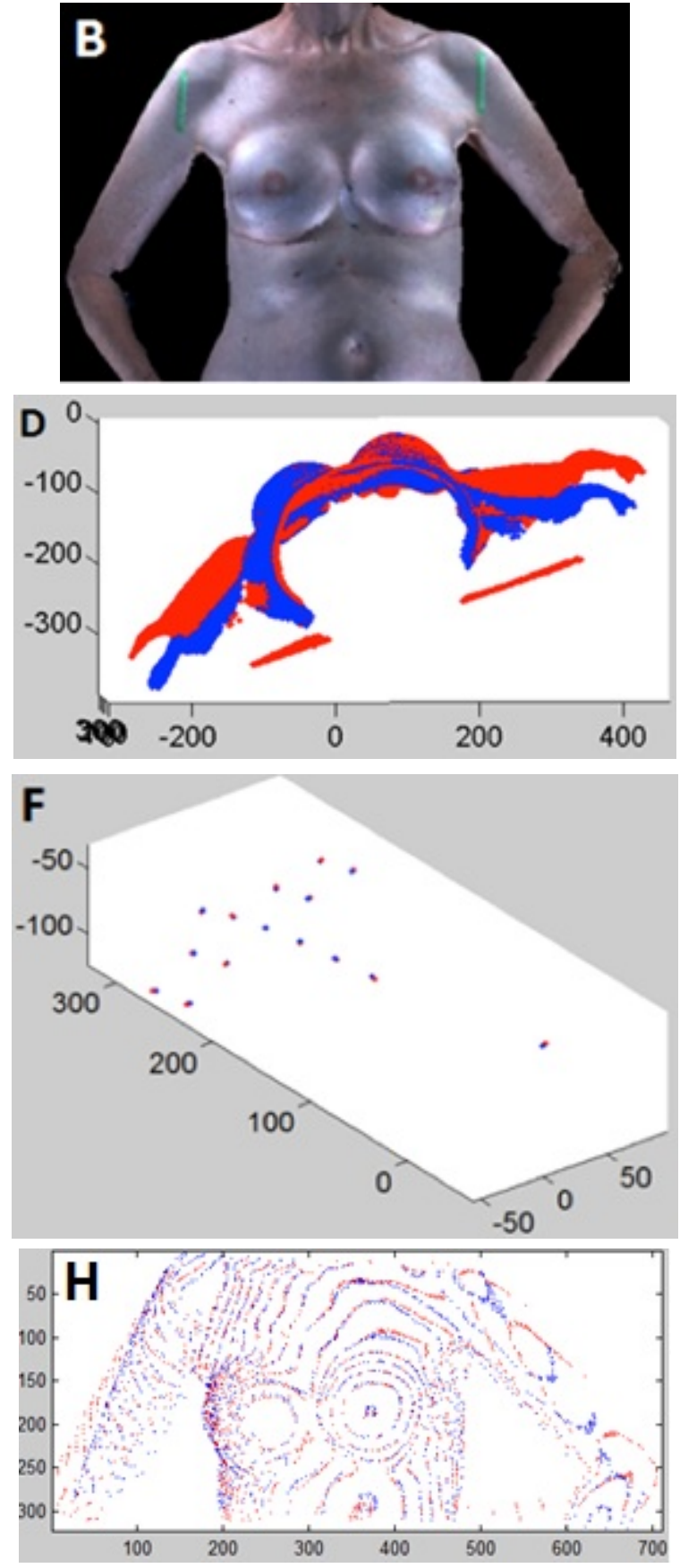

Fig. 8. Algorithm evaluation using patient images from multiple visits (visit1 in red, visit2 in blue): (A) Upright and forward facing view of $3 D$ torso from visit1, (B) Upright and forward facing view of $3 D$ torso from visit2, (C) Original point clouds (bottom-up-view of breasts) showing unmatched surfaces, (D) 3D correspondence achieved following rigid transformation using the proposed algorithm, (E) Original fiducial points (SN and UM) and the 13 reference points, (F) 3D correspondence achieved for fiducial points and selected points after registration, (G) $2 D$ point fingerprints for original surfaces, $(H) 2 D$ point fingerprint for surfaces after registration. 

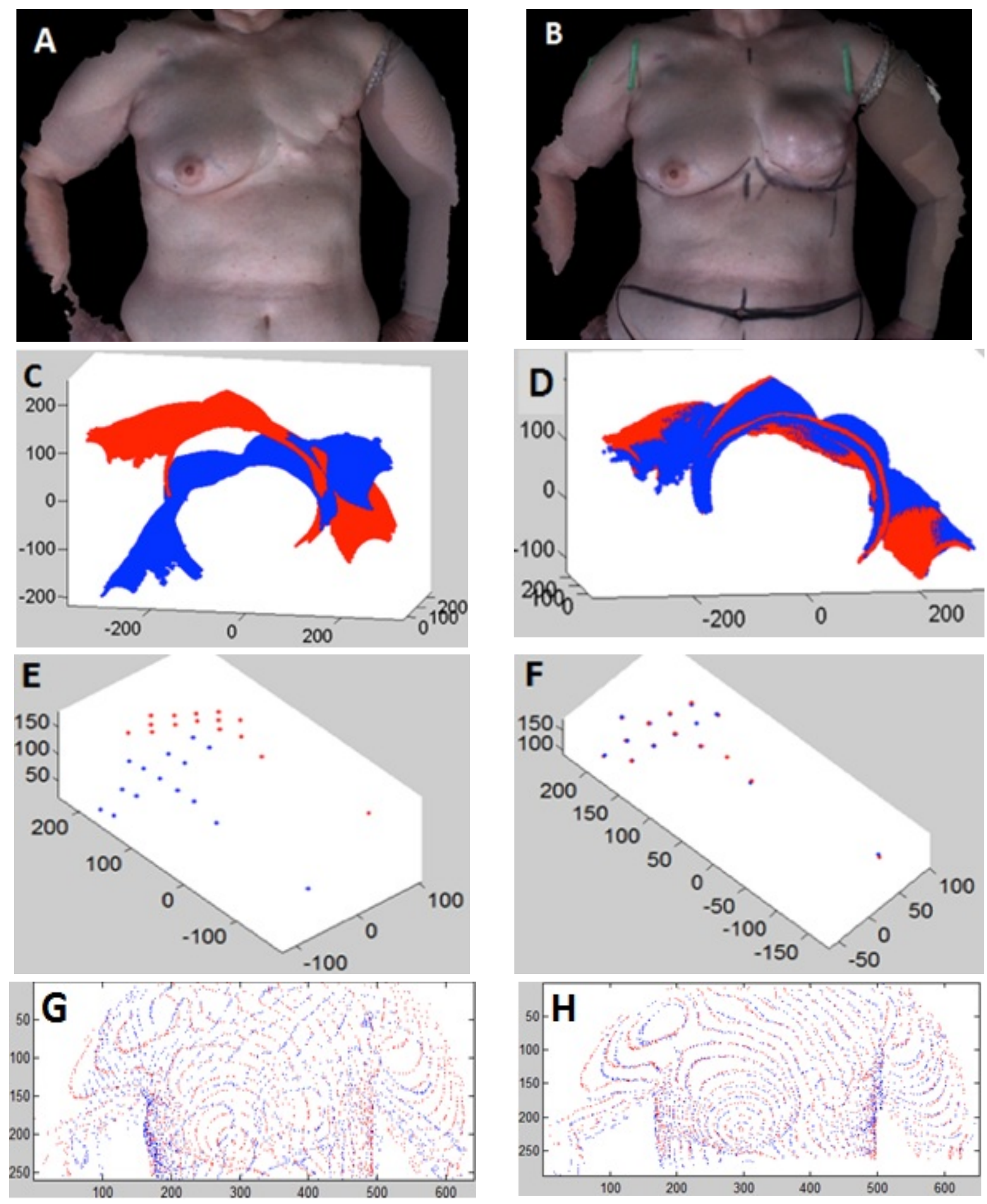

Fig. 9. Algorithm evaluation using patient images from multiple visits (visit1 in red, visit2 in blue): (A) Upright and forward facing view of $3 D$ torso from visit1, (B) Upright and forward facing view of 3D torso from visit2, (C) Original point clouds (bottom-up-view of breasts) showing unmatched surfaces, (D) 3D correspondence achieved following rigid transformation using the proposed algorithm, (E) Original fiducial points (SN and UM) and the 13 reference points, $(F) 3 D$ correspondence achieved for fiducial points and selected points after registration, (G) $2 D$ point fingerprints for original surfaces, $(H) 2 D$ point fingerprint for surfaces after registration. 


\section{References}

1. Bostwick, J. I., (1999): "Plastic and reconstructive breast surgery", 2nd Ed., St. Louis, Missouri: Quality Medical Publishing, Inc.

2. Eric, M., Mihic, N., Krivokuca, D., (2009): "Breast reconstruction following mastectomy; patient's satisfaction", Acta Chir Belg, Vol. 109, No. 2, pp. 159-166.

3. Chen, D., Chittajallu, D. R., Passalis, G., Kakadiaris, I. A., (2010): "Computational tools for quantitative breast morphometry based on 3D scans", Annals of Biomedical Engineering, Vol. 38, No. 5 , pp. 1703-1718.

4. Kawale, M., Lee, J., Leung, S. Y., Fingeret, M. C., Reece, G. P., Crosby, M. A., Beahm, E. K., Markey, M. K., Merchant, F. A., (2011): "3D symmetry measure invariant to subject pose during image acquisition", Breast Cancer: Basic and Clinical Research, Issue 5, pp. 131-142.

5. Kovacs, L., Eder, M., Hollweck, R., Zimmermann, A., Settles, M., Schneider, A., Udosic, K., Schwenzer-Zimmerer, K., Papadopulos, N. A., Biemer, E., (2006): "New aspects of breast volume measurement using 3-dimensional surface imaging", Annals of Plastic Surgery, Vol. 57, No. 6, pp. 602-610.

6. Lee, H. Y., Hong, K., Kim, E. A., (2004): "Measurement protocol of women's nude breasts using a 3D scanning technique", Applied Ergonomics, Vol. 35, No. 4, pp. 353-359.

7. Losken, A., Fishman, I., Denson, D. D., Moyer, H. R., Carlson, G. W., (2005): "An objective evaluation of breast symmetry and shape differences using 3-dimensional images", Annals Plastic Surgery, Vol. 55, No. 6, pp. 571-575.

8. Crum, W. R., Hartkens, T., Hill, D. L. G., (2004): "Non-rigid image registration: theory and practice", British Journal of Radiology, Vol. 77, Supplement 2, pp. S140-S153.

9. Wyawahare, M. V., Patil, P. M., Abhyankar, H. K., (2009): "Image registration techniques: an overview", International Journal of Signal Processing, Image Processing and Pattern Recognition, Vol. 2, No. 3, pp. 11-28.

10. Besl, P. J., McKay, N. D., Yokoya, N., (1992): "A method for registration of 3-D shapes", IEEE Trans. Pattern Analysis Machine intelligence, Vol. 14, No. 2, pp. 239-256.

11. Dorai, C., Wang, G., Jain, A. K., Mercer, C., (1998): "Registration and integration of multiple object views for 3D model construction", IEEE Trans, Pattern Anal. Machine Intelligence, Vol. 20, No. 1, pp. 83-89.

12. Masuda, T. and Yokoya, N., (1995): "A robust method for registration and segmentation of multiple range images", Computer Vision and Image Understanding, Vol. 61, No. 3, pp. 295-307.

13. Blais, G. and Levine, M. D., (1995): "Registering multiview range data to create 3D computer objects", IEEE Trans. Pattern Analysis and Machine Intelligence, Vol. 17, No. 8, pp. 820-824.

14. Masuda, T., Sakaue, K., Yokoya, N., (1996): "Registration and integration of multiple range images for 3D model construction", Proceedings of 13th International Conference on Pattern Recognition 1996, Vienna, Austria, Vol. 1, pp. 879-883.

15. Sun, Y., Paik, J., Koschan, A., Page, D., Abidi, M., (2003): "Point fingerprint: a new 3-D object representation scheme", Systems, Man and Cybernetics, Part B, IEEE Transactions on Vol. 33, No. 4, pp. 712-717.

16. Zou, G., Hua, j., Dong, M., Qin, H., (2008): "Surface matching with salient keypoints in geodesic scale space", Journal of Computer Animation and Virtual Worlds (formerly known as Journal of Visualization and Computer Animation), Vol. 19, pp. 399-410.

17. Wen, P., (2008): "Medical image registration based-on points, contour and curves", International Conference on Biomedical Engineering and Informatics 2008, Sanya, Hainan, China, pp. 132-136.

18. Zeng, Y., Wang, C., Wang, Y., Gu, X., Samaras, D., Paragios, D., (2010): "Dense non-rigid surface registration using high-order graph matching", IEEE Computer Society Conference on Computer Vision and Pattern Recognition 2010, San Francisco, CA, USA, pp. 382-389.

19. Giloi, W. K., Encarnação, J. L., Straßer, (2001): "Computer graphics pioneers: the Giloi's school of computer graphics", ACM Computer Graphics, Vol. 35, No. 4, pp. 12-16. 